Mathematical Modelling AND ANALysis

Volume 17 Number 2, April 2012, 281-292

http://dx.doi.org/10.3846/13926292.2012.664571

(C) Vilnius Gediminas Technical University, 2012
Publisher: Taylor\&Francis and VGTU

http://www.tandfonline.com/TMMA

Print ISSN: 1392-6292

Online ISSN: 1648-3510

\title{
Computing the Asymptotic Expansion of the Median of the Erlang Distribution*
}

\section{Pedro Jodrá}

\author{
Universidad de Zaragoza \\ María de Luna 3, 50018 Zaragoza, Spain \\ E-mail: pjodra@unizar.es
}

Received October 18, 2011; revised February 2, 2012; published online April 1, 2012

\begin{abstract}
We describe an algorithm for computing a large number of coefficients in the asymptotic expansion of the median of the Erlang distribution. In particular, in this paper we present the values of the first sixty coefficients which allow us to assess the importance of the higher-order terms in the behavior of the partial sums of that asymptotic expansion. As a consequence, we provide tight bounds for the median of the Erlang distribution and we also see that a conjecture concerning the complete monotonicity of a sequence of medians of the Erlang distributions is supported by numerical results.
\end{abstract}

Keywords: Erlang distribution, median, asymptotic expansion, algorithm.

AMS Subject Classification: 41A60; 60E05; 33F10.

\section{Introduction}

For any $n=0,1,2, \ldots$, let $X_{n}$ be a random variable having the Erlang distribution with shape parameter $n+1$ and scale parameter 1 , that is, its cumulative distribution function $F_{n}(x):=P\left(X_{n} \leq x\right)$ is given by

$$
F_{n}(x)=\frac{1}{n !} \int_{0}^{x} t^{n} e^{-t} d t, \quad x \geq 0 .
$$

The median of $X_{n}$, hereafter denoted by $\lambda_{n}$, is defined as the unique solution of the equation $F_{n}\left(\lambda_{n}\right)=1 / 2$. Analytical solutions of this equation are known only for the simplest cases $n=0$ and $n=1$. For the former case, it is trivial that $\lambda_{0}=\log 2$, and, for the latter case, Jiménez and Jodrá [11] have shown that $\lambda_{1}=-W_{-1}(-1 / 2 e)-1$, where $W_{-1}$ denotes the negative branch of the Lambert $W$ function. Accordingly, over the last two decades several authors have given upper and lower bounds for $\lambda_{n}$, instead of analytical solutions,

* This work has been supported by DGA (Grupo consolidado PDIE) and by Vicerrectorado de Investigación (Universidad de Zaragoza), research project 229/128. 
and, in this respect, tight bounds for $\lambda_{n}$ have been obtained by considering appropriate partial sums of the asymptotic expansion of $\lambda_{n}$.

In 1986, Chen and Rubin [7] conjectured the following bounds for the median of $X_{n}: n+2 / 3<\lambda_{n} \leq n+\log 2$, for $n=0,1,2, \ldots$ Choi [9] proved this conjecture by showing that

$$
n+\frac{2}{3}<\lambda_{n} \leq \min \left(n+\log 2, n+\frac{2}{3}+\frac{1}{2 n+2}\right), \quad n=0,1,2, \ldots
$$

These bounds were subsequently improved by Adell and Jodrá [1], namely,

$$
n+\frac{2}{3}+\frac{8}{405 n}-\frac{64}{5103 n^{2}}<\lambda_{n}<n+\frac{2}{3}+\frac{8}{405 n}, \quad n=1,2, \ldots
$$

It is interesting to note that the bounds in Eq. (1.1) are partial sums of the asymptotic expansion of $\lambda_{n}$, and, in this regard, Choi [9] deduced the first four coefficients in that expansion

$$
\lambda_{n}=n+\frac{2}{3}+\frac{8}{405 n}-\frac{64}{5103 n^{2}}+\frac{2944}{492075 n^{3}}+O\left(\frac{1}{n^{4}}\right) .
$$

Throughout this paper, for integers $j \geq 0$ we denote by $q_{j}$ the coefficient of $n^{-j}$ in the asymptotic expansion of $\lambda_{n}$, i.e., $\lambda_{n}=n+\sum_{j=0}^{\infty} q_{j} / n^{j}$, where it is understood that $q_{0}:=2 / 3$.

In order to obtain formula (1.2), Choi established a relation between the median of $X_{n}$ and a sequence $\left\{\theta_{n}\right\}_{n \geq 0}$ introduced by Ramanujan [13] in 1911 (cf. also Ramanujan [14]) and defined as the solution of the equation

$$
\theta_{n}=\frac{n !}{n^{n}}\left(\frac{e^{n}}{2}-\sum_{k=0}^{n-1} \frac{n^{k}}{k !}\right), \quad n=1,2, \ldots,
$$

and setting $\theta_{0}=1 / 2$. More precisely, Choi [9] pointed out the following relation between $\theta_{n}$ and $\lambda_{n}$

$$
1-\theta_{n}=\frac{e^{n}}{n^{n}} \int_{n}^{\lambda_{n}} t^{n} e^{-t} d t, \quad n=1,2, \ldots,
$$

and he deduced formula (1.2) by considering an asymptotic expansion of $\theta_{n}$ on the left-hand side of Eq. (1.3). Asymptotic expansions of $\theta_{n}$ have been obtained by several authors, beginning with Ramanujan [13] who gave the first four terms

$$
\theta_{n}=\frac{1}{3}+\frac{4}{135 n}-\frac{8}{2835 n^{2}}-\frac{16}{8505 n^{3}}+O\left(\frac{1}{n^{4}}\right)
$$

In the middle 1980s, Bowman et al. [6] found the 5th and 6th coefficients and finally Marsaglia [12] developed a numerically stable procedure to compute a large number of coefficients in that asymptotic expansion.

With the aim of refining the bounds given in Eq. (1.1), Adell and Jodrá [2] computed the first seven coefficients $q_{j}$ in the asymptotic expansion of $\lambda_{n}$ (see Table 1) and then they derived the following upper and lower bounds for $\lambda_{n}$ : 
$n+2 / 3+\sum_{j=1}^{6} q_{j} / n^{j}<\lambda_{n}<n+2 / 3+\sum_{j=1}^{7} q_{j} / n^{j}$, for $n=1,2 \ldots$ In addition, they considered that sharper bounds for $\lambda_{n}$ can be obtained in a similar way by considering more terms in the asymptotic expansion of $\lambda_{n}$. But, as we can see in Table 1 , the coefficients $q_{j}$ have a value very close to 0 for $j=5,6$ and 7 , so that if the values of the $q_{j}$ 's are also very close to 0 for all $j \geq 8$ it is not clear that sharper bounds for $\lambda_{n}$, valid for all positive integers $n$, can be successively obtained by considering more terms in that asymptotic expansion.

Table 1. Coefficients $q_{j}, j=1, \ldots, 7$.

\begin{tabular}{rlr}
\hline$j$ & $q_{j}$ & \\
\hline 1 & $\frac{8}{405}$ & $\approx 0.019753086419$ \\
2 & $-\frac{64}{5103}$ & $\approx-0.012541642171$ \\
3 & $\frac{2944}{492075}$ & $\approx 0.005982827820$ \\
4 & $-\frac{20182528}{15345358875}$ & $\approx-0.001315220332$ \\
5 & $-\frac{8465604608}{12567848918625}$ & $\approx-0.000673592168$ \\
6 & $\frac{527611584512}{166665960014375}$ & $\approx 0.000310970794$ \\
7 & $\frac{101756461251198976}{136284182692454671875}$ & $\approx 0.000746649091$ \\
\hline
\end{tabular}

In view of the above considerations, in this paper we pose the following questions:

(i) Does the sequence $\left\{\left|q_{j}\right|\right\}_{j \geq 1}$ converge to 0 such as the numerical results in Table 1 may be suggesting $(|\cdot|$ denotes the absolute value)?

(ii) Which is the partial sum of the asymptotic expansion of $\lambda_{n}$ closest to the true value of $\lambda_{n}$ as well as its number of significant digits?

(iii) Can sharper bounds for $\lambda_{n}$ be obtained by considering more terms in the asymptotic expansion of $\lambda_{n}$ as it is proposed in Adell and Jodrá [2]?

Unfortunately, as far as we know, the coefficients $q_{j}$ cannot be expressed in closed form. In this paper, we study the above questions from a numerical point of view and, to this end, first we need to compute more coefficients in the asymptotic expansion of $\lambda_{n}$. In Section 2, we develop an algorithm for computing a large number of coefficients in the asymptotic expansion of $\lambda_{n}$. In Section 3, we present the numerical results obtained and, in particular, we give tight upper and lower bounds for $\lambda_{n}$. Finally, we see that a conjecture proposed by Alzer [4] concerning the complete monotonicity of the sequence $\left\{\lambda_{n}-n\right\}_{n \geq 0}$ is supported by numerical results.

\section{An Algorithm for Computing the Asymptotic Expan- sion of the Median}

In this section, we present an algorithm for computing the asymptotic expansion of $\lambda_{n}$ which is an improved version of Choi's procedure. First, we briefly 
describe the method outlined by Choi [9]. From Eq. (1.3), Choi showed the following relationship between $\theta_{n}$ and $\lambda_{n}$

$$
\theta_{n}-1=\sum_{k=0}^{\infty} \frac{c_{k}(n)}{(k+1) !}\left(n-\lambda_{n}\right)^{k+1}, \quad n=1,2, \ldots,
$$

where the coefficients $c_{k}(n)$ satisfy the recurrence relation $n c_{k}(n) /(1-k)=$ $\left(c_{k-1}(n)+c_{k-2}(n)\right)$, for integers $k \geq 2$, with initial conditions $c_{0}(n):=1$ and $c_{1}(n):=0$. By considering Eq. (2.1), Choi proposed to compute the coefficients in the asymptotic expansion of $\lambda_{n}$ as follows. On the left-hand side of Eq. (2.1) it is considered the asymptotic expansion of $\theta_{n}$, and, on the right-hand side of Eq. (2.1) the series involved is decomposed into a partial fraction expansion of terms of order $n^{-j}$, with $j \geq 0$. After that decomposition step, the coefficients corresponding to each term of order $n^{-j}$ in both sides of Eq. (2.1) are equated and thereby the $q_{j}$ 's in the asymptotic expansion of $\lambda_{n}$ can be obtained.

In addition, Adell and Jodrá [2] noted that the terms $c_{k}(n)$ in Eq. (2.1) are Charlier polynomials. We recall that Charlier polynomials can be explicitly defined by (cf. Chihara [8, Chap. VI])

$$
C_{k}(t ; n):=\sum_{j=0}^{k}\left(\begin{array}{l}
k \\
j
\end{array}\right)\left(\begin{array}{l}
n \\
j
\end{array}\right) j !(-t)^{-j}, \quad k=0,1, \ldots,
$$

where $t>0$ and $n=0,1, \ldots$ More specifically, Adell and Jodrá [2] noticed that $c_{k}(n)=C_{k}(n ; n)$ so that the coefficients $c_{k}(n)$ in Eq. (2.1) can also be computed by means of Eq. (2.2).

With the help of the symbolic computer algebra system Maple Release 12, and based on the above considerations, we have implemented the brute force algorithm outlined by Choi. We have chosen Maple because it yields a suitable fraction decomposition of the right-hand side of Eq. (2.1) as a sum of terms of order $n^{-j}$, with $j \geq 0$. However, the running time and space required increases dramatically and only a few coefficients in the asymptotic expansion of $\lambda_{n}$ can be computed.

Table 2. CPU time used for computing $q_{j}, j=1, \ldots, i$.

\begin{tabular}{lc}
\hline \multicolumn{2}{c}{ Brute force algorithm } \\
\hline Computing $\left\{q_{j}\right\}_{j=1}^{i}$ & CPU time (in seconds) \\
\hline$i=5$ & 1.7 \\
$i=10$ & 19.4 \\
$i=15$ & 466.1 \\
$i=19$ & 4188.5 \\
\hline
\end{tabular}

Table 2 displays the CPU time used for computing some coefficients $q_{j}$. In particular, the coefficients $q_{j}$, for $j \geq 20$, could not be obtained using the brute force algorithm due to memory limitations; Maple exceeded the amount of memory available $(2.0 \mathrm{~GB})$ and the execution of the algorithm was aborted. 
All of the computations were performed on an Intel Core2 Quad Q8200 at $2.33 \mathrm{GHz}$ with $4 \mathrm{~GB}$ RAM.

In the remainder of this section, we improve Choi's brute force algorithm. To start with, we introduce some notation. Denote by

$$
E_{i}(n):=\sum_{k=0}^{2 i} \frac{C_{k}(n ; n)}{(k+1) !}\left(n-\lambda_{i}(n)\right)^{k+1}, \quad i=1,2, \ldots,
$$

where

$$
\lambda_{i}(n):=n+\frac{2}{3}+\sum_{j=1}^{i} \frac{q_{j}}{n^{j}}, \quad i=1,2, \ldots
$$

For our purpose, we need to express the function $E_{i}(n)$ as a fraction decomposition of terms of order $n^{-j}$, with $j \geq 0$. To this end, first it can be checked from Eq. (2.2) that the Charlier polynomials $C_{k}(n ; n)$, for $k \geq 2$, can be expressed as below

$$
C_{k}(n ; n)=\sum_{j=\lceil k / 2\rceil}^{k-1} \frac{b_{j}^{(k)}}{n^{j}}, \quad k=2,3, \ldots,
$$

where the coefficients $b_{j}^{(k)}$ are rational numbers; as usual, $\lceil x\rceil$ denotes the ceiling of a real number $x$, that is, the smallest integer not less than $x$. Then, by considering Eq. (2.3) together with Eq. (2.4), it is clear that $E_{i}(n)$ can be expressed as follows

$$
E_{i}(n)=\sum_{j=0}^{2 i^{2}+3 i-1} \frac{\beta_{j}^{(i)}}{n^{j}}, \quad i=1,2, \ldots,
$$

where the coefficients $\beta_{j}^{(i)}$ are rational numbers. Unfortunately, we do not know a closed-form expression for computing the coefficients $\beta_{j}^{(i)}$ in Eq. (2.5).

In addition to the previous notation, throughout this paper we also consider $E_{0}(n):=-2 / 3, \beta_{0}^{(0)}:=-2 / 3, \lambda_{0}(n):=n+2 / 3$ and, finally, we assume that $b_{1}^{(1)}:=0$ since $C_{1}(n ; n)=0$.

The algorithm that we propose is sequential so that $q_{i}$ is calculated only after $q_{1}, \ldots, q_{i-1}$ have been obtained. For any integer $i \geq 1$, it can be checked that all of the summands on the right-hand side of Eq. (2.3) are meaningful to compute $q_{i}$ because each one of these summands contributes to the finite sum on the right-hand side of Eq. (2.5) with terms of order $n^{-j}$ for some $j \leq i$. In order to compute $q_{1}, \ldots, q_{i}$ we shall need to compute successively the functions $E_{1}(n), \ldots, E_{i}(n)$ by means of formula (2.3), which implies a large computational effort, and to obtain these functions in a more efficient way we derive the following relation.

Theorem 1. For any integer $n \geq 1$ and $i=1,2, \ldots$, we have

$$
\begin{aligned}
E_{i}(n)= & E_{i-1}(n)+\frac{C_{2 i-1}(n ; n)}{(2 i) !}\left(n-\lambda_{i-1}(n)\right)^{2 i}+\frac{C_{2 i}(n ; n)}{(2 i+1) !}\left(n-\lambda_{i-1}(n)\right)^{2 i+1} \\
& +\sum_{k=0}^{2 i} \sum_{r=1}^{k+1}(-1)^{r}\left(\begin{array}{c}
k+1 \\
r
\end{array}\right) \frac{C_{k}(n ; n)}{(k+1) !}\left(n-\lambda_{i-1}(n)\right)^{k+1-r}\left(\frac{q_{i}}{n^{i}}\right)^{r},
\end{aligned}
$$


with initial conditions $E_{0}(n):=-2 / 3$ and $\lambda_{0}(n):=n+2 / 3$.

Proof. From Eq. (2.3) and taking into account that $\lambda_{i}(n)=\lambda_{i-1}(n)+q_{i} / n^{i}$, we can write $E_{i}(n)$ as follows

$$
\begin{aligned}
& E_{i}(n)=\sum_{k=0}^{2(i-1)} \frac{C_{k}(n ; n)}{(k+1) !}\left(n-\lambda_{i-1}(n)-q_{i} / n^{i}\right)^{k+1} \\
& +\frac{C_{2 i-1}(n ; n)}{(2 i) !}\left(n-\lambda_{i-1}(n)-q_{i} / n^{i}\right)^{2 i}+\frac{C_{2 i}(n ; n)}{(2 i+1) !}\left(n-\lambda_{i-1}(n)-q_{i} / n^{i}\right)^{2 i+1} .
\end{aligned}
$$

Moreover, by Newton's binomial formula, for $j=1,2, \ldots$ we know

$$
\left(n-\lambda_{i-1}(n)-q_{i} / n^{i}\right)^{j}=\sum_{r=0}^{j}\left(\begin{array}{l}
j \\
r
\end{array}\right)\left(n-\lambda_{i-1}(n)\right)^{j-r}\left(-\frac{q_{i}}{n^{i}}\right)^{r} .
$$

The statement of the theorem readily follows by considering formula (2.8) into Eq. (2.7) and a bit of algebra. The proof is complete.

From a computational point of view, Eq. (2.6) highlights the large amount of calculations required to obtain $E_{i}(n)$ even when $E_{i-1}(n)$ has been previously computed. We also remark that the coefficient $q_{i}$ appears explicitly only in the last term of Eq. (2.6) and it is easy to see that $q_{i}$ contributes to the value of $\beta_{j}^{(i)}$ in Eq. (2.5) only for $j \geq i$. In addition, as we see in the following result, the values of the coefficients $\beta_{j}^{(i)}$ are known a priori for $j=0,1, \ldots, i$. Denote by $c_{j}$ the coefficient of $n^{-j}$ in the asymptotic expansion of $\left(\theta_{n}-1\right)$, that is, $\theta_{n}-1=\sum_{j=0}^{\infty} c_{j} / n^{j}$. It is interesting to recall that a large number of coefficients $c_{j}$ can be efficiently computed by using the algorithm proposed by Marsaglia [12].

Corollary 1. For any integer $i \geq 1$, we have $\beta_{j}^{(i)}=c_{j}$ for $j=0,1, \ldots, i$.

Proof. For any integer $i \geq 1$, from the relation between $\theta_{n}$ and $\lambda_{n}$ given in Eq. (2.1) and taking into account Eq. (2.3) together with the asymptotic expansion of $\left(\theta_{n}-1\right)$ and Theorem 1 , we get

$$
\sum_{j=0}^{i} c_{j} / n^{j}=\sum_{j=0}^{i} \beta_{j}^{(i)} / n^{j}
$$

which implies the result.

On the other hand, the value of each coefficient $\beta_{j}^{(i)}$ in Eq. (2.5), for $j=$ $i+1, \ldots, 2 i^{2}+3 i-1$, can be calculated by virtue of Theorem 1 as we see below. Before we introduce a more compact notation for the last term in Eq. (2.6). For integers $n \geq 1$ and $i \geq 1$, and $k=0,1, \ldots, 2 i$, denote by

$$
\begin{aligned}
& D_{i}(k, r ; n):=(-1)^{r}\left(\begin{array}{c}
k+1 \\
r
\end{array}\right) \frac{C_{k}(n ; n)}{(k+1) !}\left(n-\lambda_{i-1}(n)\right)^{k+1-r}\left(\frac{q_{i}}{n^{i}}\right)^{r}, \\
& \quad r=1, \ldots, k+1 .
\end{aligned}
$$


Denote by $\left[n^{j}\right] f(n)$ the coefficient of $n^{-j}$ in a function $f(n)=\sum_{j \geq 0} s_{j} / n^{j}$. With the preceding notations, we state the following.

Corollary 2. For any integer $i \geq 1$, we have

$$
\begin{aligned}
& \beta_{j}^{(i)}=\beta_{j}^{(i-1)}+\left[n^{j}\right] \frac{C_{2 i-1}(n ; n)}{(2 i) !}\left(n-\lambda_{i-1}(n)\right)^{2 i}+\left[n^{j}\right] \frac{C_{2 i}(n ; n)}{(2 i+1) !} \\
& \times\left(n-\lambda_{i-1}(n)\right)^{2 i+1}+\sum_{k=0}^{2 i} \sum_{r=1}^{k+1}\left[n^{j}\right] D_{i}(k, r ; n), \quad j=i+1, \ldots, 2 i^{2}+3 i-1,
\end{aligned}
$$

with initial condition $\beta_{j}^{(0)}:=0$ for $j=2,3$ and 4 .

Finally, below we give a formula to obtain the value of $q_{i}$ assuming that the coefficients $q_{1}, \ldots, q_{i-1}$ have been previously computed and, therefore, the value of $\beta_{i}^{(i-1)}$ has also been obtained by virtue of Corollary 2 .

Corollary 3. For any integer $i \geq 1$, we have

$$
q_{i}=\beta_{i}^{(i-1)}+\left(\frac{2}{3}\right)^{2 i} \frac{1}{(2 i) !} b_{i}^{(2 i-1)}-\left(\frac{2}{3}\right)^{2 i+1} \frac{1}{(2 i+1) !} b_{i}^{(2 i)}-c_{i},
$$

where it is assumed that $\beta_{1}^{(0)}:=0$.

Proof. Let us now consider Eq. (2.6). For any integer $i \geq 1$, by taking into account Eq. (2.4), it can be checked the following

$$
\begin{aligned}
& {\left[n^{i}\right] \frac{C_{2 i-1}(n ; n)}{(2 i) !}\left(n-\lambda_{i-1}(n)\right)^{2 i}=\left(\frac{2}{3}\right)^{2 i} \frac{1}{(2 i) !} b_{i}^{(2 i-1)},} \\
& {\left[n^{i}\right] \frac{C_{2 i}(n ; n)}{(2 i+1) !}\left(n-\lambda_{i-1}(n)\right)^{2 i+1}=-\left(\frac{2}{3}\right)^{2 i+1} \frac{1}{(2 i+1) !} b_{i}^{(2 i)},}
\end{aligned}
$$

and it is also clear that $\sum_{k=0}^{2 i} \sum_{r=1}^{k+1}\left[n^{i}\right] D_{i}(k, r ; n)=-q_{i}$. Then, from Eq. (2.6) we have

$$
\left[n^{i}\right] E_{i}(n)=\beta_{i}^{(i-1)}+\left(\frac{2}{3}\right)^{2 i} \frac{1}{(2 i) !} b_{i}^{(2 i-1)}-\left(\frac{2}{3}\right)^{2 i+1} \frac{1}{(2 i+1) !} b_{i}^{(2 i)}-q_{i}
$$

and, on the other hand, from Corollary 1 we also know that $\beta_{i}^{(i)}=c_{i}$, which implies the result.

Next, we give a brief description of the algorithm. In order to compute the first $m$ coefficients $q_{1}, \ldots, q_{m}$, first, in a preprocessing step, the coefficients $c_{j}$, for $j=1, \ldots, m$, are computed using the algorithm given by Marsaglia [12]. Moreover, the coefficients $b_{j}^{(2 j-1)}$ and $b_{j}^{(2 j)}$, for $j=1,2, \ldots, m$, can also be computed from Eq. (2.2) in a preprocessing step. Now, let us consider a step $i \leq m$ of the algorithm and let us assume that $q_{1}, \ldots, q_{i-1}$ together with $\beta_{j}^{(i-1)}$, for $j=i, \ldots, m$, are known. Then, the coefficient $q_{i}$ can be calculated by virtue 
of Corollary 3. In the case $i=m, \lambda_{m}(n)$ has been obtained and the algorithm terminates. On the contrary, in the case $i<m$, after computing the coefficient $q_{i}$ we calculate the value of $\beta_{j}^{(i)}$, for $j=i+1, \ldots, m$, according to Corollary 2 . It must also be noted that the coefficients $\beta_{j}^{(i)}$, for $j=m+1, \ldots, 2 i^{2}+3 i-1$, can be disregarded if we calculate only the first $m$ coefficients $q_{1}, \ldots, q_{m}$. In this respect, an additional simplification reduces significantly the running time and space requirements of the algorithm. For $k=0, \ldots, 2 i$, it can be checked that each term $D_{i}(k, r ; n), r=1, \ldots, k+1$, in Eq. (2.9) can be removed if $i r+\lceil k / 2\rceil>m$ because, in this case, the fraction decomposition of $D_{i}(k, r ; n)$ contains only terms of order $n^{-j}$ for some $j>m$.

Remark 1 . In order to compute the first $m$ coefficients $q_{1}, \ldots, q_{m}$ in the asymptotic expansion of $\lambda_{n}$, in each step $i<m$ of the algorithm we have to calculate and store only the coefficients $\beta_{j}^{(i)}$ for $j=i+1, \ldots, m$. In particular, this implies that for $k=0, \ldots, 2 i$, each term $D_{i}(k, r ; n), r=1, \ldots, k+1$, in Eq. (2.9) can be disregarded if $i r+\lceil k / 2\rceil>m$.

As a summary, Figure 1 below shows a way of doing the computations in a pseudocode.

We have implemented in Maple Release 12 the algorithm described in this section. With the help of Maple, each function involved in Theorem 1 can

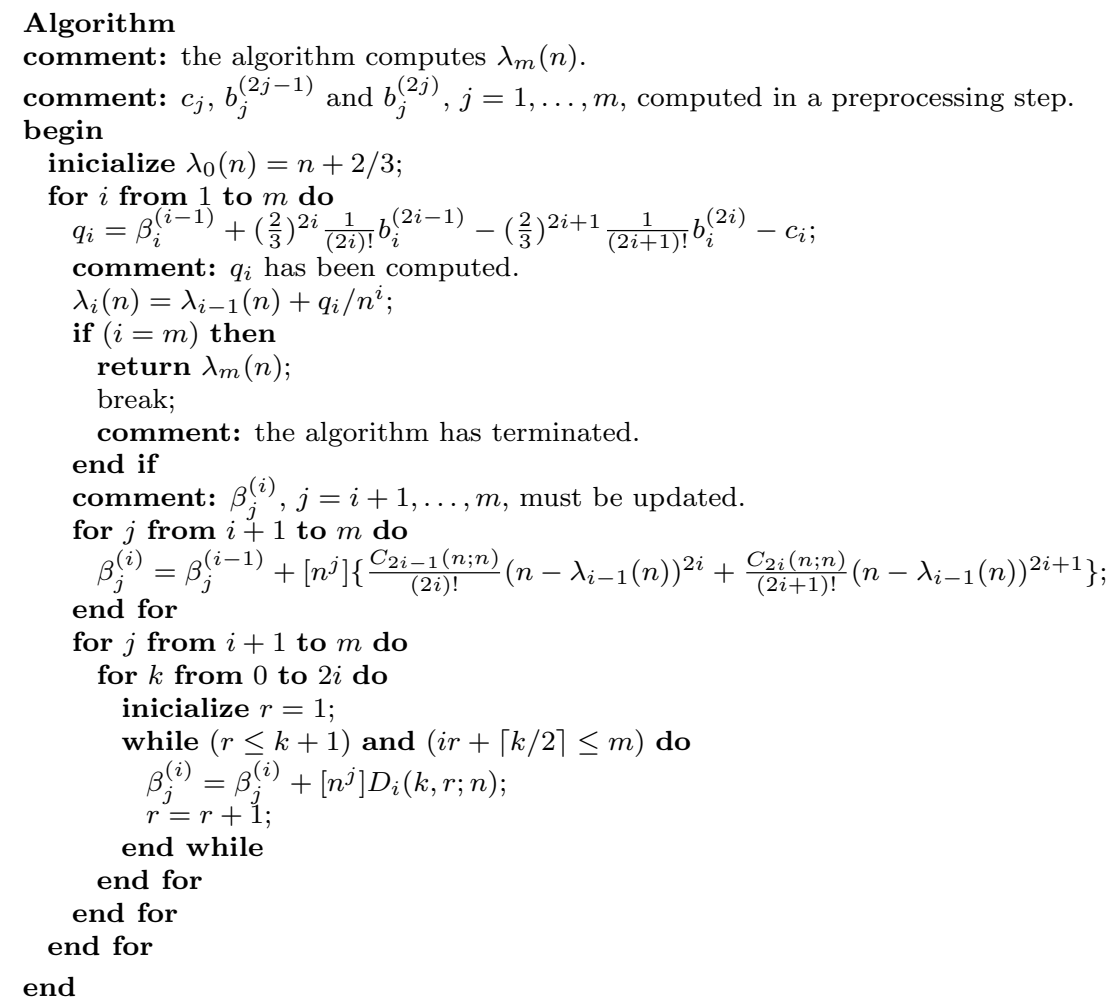

Figure 1. Algorithm for computing $\lambda_{m}(n)$. 
Table 3. The rational coefficients $q_{i}, i=1, \ldots, 20$, and their approximate values.

\begin{tabular}{|c|c|c|}
\hline$i$ & $q$ & \\
\hline 1 & $\frac{8}{405}$ & $\approx 0.019753086419$ \\
\hline 2 & $-\frac{64}{5103}$ & $\approx-0.012541642171$ \\
\hline 3 & $\frac{2944}{492075}$ & $\approx 0.005982827820$ \\
\hline 4 & $-\frac{20182528}{15345358875}$ & $\approx-0.001315220332$ \\
\hline 5 & $-\frac{8465604608}{12567848918625}$ & $\approx-0.000673592168$ \\
\hline 6 & $\frac{527611584512}{1696659604014375}$ & 0.000310970794 \\
\hline 7 & $\frac{101756461251198976}{136284182692454671875}$ & 0.000746649092 \\
\hline 8 & $-\frac{1091865798741131264}{2118599567309977171875}$ & $\approx-0.000515371482$ \\
\hline 9 & $-\frac{113445250854901086814208}{103821971796025431307734375}$ & $\approx-0.001092690197$ \\
\hline 10 & $\frac{188986533236068276352319488}{173582350537431749959508203125}$ & 0.001088742793 \\
\hline 11 & $\frac{140359054398115632025068634112}{52803751033486738337682395390625}$ & 0.002658126584 \\
\hline 12 & $-\frac{122834986466715962736247558897664}{35642531947603548377935616888671875}$ & $\approx-0.003446303608$ \\
\hline 13 & $-\frac{910021020968802126714213584465228201984}{96701575214383449086435232502571220703125}$ & $\approx-0.009410612174$ \\
\hline 14 & $\frac{407082682842868086242134902527542918381568}{26979739484812982295115429868217370576171875}$ & 0.015088458621 \\
\hline 15 & $\frac{99080521411381459617898992340510464422132580352}{21671475741117602802855146904164560291531005859375}$ & 0.045719323683 \\
\hline 16 & $-\frac{47521101500531306640850145040380162843845159026688}{543334856082341845572968973829829044519559326171875}$ & $\approx-0.087461904879$ \\
\hline 17 & $-\frac{343842430576068287990570735916021205705305145050574880768}{1179124114610511061930479921215515629083611386844482421875}$ & $\approx-0.291608344122$ \\
\hline 18 & $\frac{3407806529052351556350031552321433178204905245704385239973888}{5252997930589826780900288049015122127567488728392169189453125}$ & 0.648735555216 \\
\hline 19 & $\frac{6243991993888021860360669674760577685646172219659776673313718272}{2643213049619517384753008577390790997826004555604605133056640625}$ & 2.362273443976 \\
\hline 20 & $-\frac{472179990608599254283064265692787651720998971554386635000540924215296}{78765105665611998548254902597668180944217109752461628359954833984375}$ & $\approx-5.994786480871$ \\
\hline
\end{tabular}

be decomposed into a partial fraction of terms of order $n^{-j}$, with $j \geq 0$. In particular, we have used some functions provided in the Maple-package PolynomialTools (cf. Heck [10, Chap. 5]). As a result, we have obtained exactly, that is, as rational numbers, a large number of coefficients in the asymptotic expansion of $\lambda_{n}$. In this paper we present only the first sixty coefficients and, as numerators and denominators become large, for the sake of space we display the first twenty rational coefficients in Table 3, which were computed in less than 24.5 seconds CPU time, and approximate values for the other coefficients in Table 4.

\section{Numerical Consequences}

At the end of Section 1 three questions were posed. As analytical expressions for the coefficients in the asymptotic expansion of $\lambda_{n}$ have not been found, we use the numerical results obtained in the previous section to answer those questions.

To answer question (i) concerning the convergence to 0 of the sequence $\left\{\left|q_{i}\right|\right\}_{i \geq 1}$, from Tables 3 and 4 we see that the value of $\left|q_{i}\right|$ strictly increases as $i$ increases for $i \geq 10$. Thereby, based on the numerical evidence, we assert that the sequence $\left\{\left|q_{i}\right|\right\}_{i \geq 1}$ does not converge to 0 , on the contrary, we conjecture that $\left\{\left|q_{i}\right|\right\}_{i \geq 1}$ diverges. 
Table 4. The approximate value of $q_{i}$, for $i=21, \ldots, 60$.

\begin{tabular}{rr|rr}
\hline$i$ & \multicolumn{1}{c|}{$q_{i}$} & \multicolumn{1}{l}{$i$} & $q_{i}$ \\
\hline 21 & -23.684361348669 & 41 & $-1.1564885959677822668958501234319231 \cdot 10^{15}$ \\
22 & 67.5418513427755 & 42 & $6.9561716365916852165241531460296780 \cdot 10^{15}$ \\
23 & 287.85895156268050 & 43 & $5.1553534410496517825406144072457824 \cdot 10^{16}$ \\
24 & -911.509961320613176 & 44 & $-3.2644933042045415863738250108794785 \cdot 10^{17}$ \\
25 & -4169.4873282691158125 & 45 & $-2.5227468509246430418663688747868782 \cdot 10^{18}$ \\
26 & 14517.51062315480054612 & 46 & $1.6775405047079434174954084653643242 \cdot 10^{19}$ \\
27 & 70954.77947196402774553 & 47 & $1.3495185248199705999283863810313058 \cdot 10^{20}$ \\
28 & $-2.69458840306955185 \cdot 10^{5}$ & 48 & $-9.4023020801550544877879262847600277 \cdot 10^{20}$ \\
29 & $-1.40159861100601650 \cdot 10^{6}$ & 49 & $-7.8617762090166445430502188919197474 \cdot 10^{21}$ \\
30 & $5.76579884987129503 \cdot 10^{6}$ & 50 & $5.7270790609799904579501055404994538 \cdot 10^{22}$ \\
31 & $3.18048234453219928 \cdot 10^{7}$ & 51 & $4.9702887257257575962402656022736164 \cdot 10^{23}$ \\
32 & $-1.40899461576200422 \cdot 10^{8}$ & 52 & $-3.7785791960159019135070330313173696 \cdot 10^{24}$ \\
33 & $-8.21609794289168550 \cdot 10^{8}$ & 53 & $-3.3990989824854793566420201466586793 \cdot 10^{25}$ \\
34 & $3.89998255563806189 \cdot 10^{9}$ & 54 & $2.6920880364957272549758657789254373 \cdot 10^{26}$ \\
35 & $2.3971739322341164 \cdot 10^{10}$ & 55 & $2.5071217823317726158976924227246943 \cdot 10^{27}$ \\
36 & $-1.2138276743182051 \cdot 10^{11}$ & 56 & $-2.0652973718779740795700093359996914 \cdot 10^{28}$ \\
37 & $-7.8442406406263566 \cdot 10^{11}$ & 57 & $-1.9889278856682013624948681734354735 \cdot 10^{29}$ \\
38 & $4.2206394905418625 \cdot 10^{12}$ & 58 & $1.7016236284887685450280925006068876 \cdot 10^{30}$ \\
39 & $2.8609375093053603 \cdot 10^{13}$ & 59 & $1.6927062244703204737287295257032487 \cdot 10^{31}$ \\
40 & $-1.6300691594774892 \cdot 10^{14}$ & 60 & $-1.5019853275923149545469547291382864 \cdot 10^{32}$ \\
\hline
\end{tabular}

To answer question (ii), that is, which is the partial sum $\lambda_{i}(n)$ of the asymptotic expansion of $\lambda_{n}$ closest to the true value of $\lambda_{n}$, for small values of $n$ we have checked that the series of partial sums $\left\{\lambda_{i}(n)\right\}_{i \geq 1}$ behaves as asymptotic series often do. The partial sums $\lambda_{i}(n)$ approach the true value of $\lambda_{n}$, reach the point of closest approach and then become increasingly worse. Table 5 shows the results obtained for a few small values of $n$. We highlight that small values of $n$ are the most frequent cases in real applications of the Erlang distribution.

Table 5. Partial sums $\lambda_{i}(n)$ approaching $\lambda_{n}$, for $n=1, \ldots, 9$.

\begin{tabular}{ccc}
\hline$n$ & $\begin{array}{c}\text { Partial sum } \\
\lambda_{i}(n) \text { closest to } \lambda_{n} \\
i\end{array}$ & $\begin{array}{c}\text { Number of correct } \\
\text { significant digits }\end{array}$ \\
\hline 1 & 10 th & 3 \\
2 & 16 th & 6 \\
3 & $22 \mathrm{nd}$ & 10 \\
4 & $28 \mathrm{th}$ & 11 \\
5 & $34 \mathrm{th}$ & 15 \\
6 & $40 \mathrm{th}$ & 17 \\
7 & $48 \mathrm{th}$ & 21 \\
8 & $54 \mathrm{th}$ & 24 \\
9 & $60 \mathrm{th}$ & 27 \\
\hline
\end{tabular}

Now, we answer question (iii) related to the bounds of the median of the Erlang distribution. As it was said in Section 1, Adell and Jodrá [2] have given upper and lower bounds for $\lambda_{n}$, specifically $\lambda_{6}(n)<\lambda_{n}<\lambda_{7}(n)$ for $n \geq 1$. Moreover, Adell and Jodrá [2, Section 4] remark that the same methodology can be used to obtain sharper bounds for $\lambda_{n}$ if we consider more terms in the 
asymptotic expansion of $\lambda_{n}$. From the numerical results presented in Tables 3 and 4 , we have assessed the importance of the higher-order coefficients in that asymptotic expansion in order to derive sharper bounds for $\lambda_{n}$ and, in this regard, we have checked that those bounds are not always valid for small values of $n$. This possibility has not been considered by Adell and Jodrá [2] and, accordingly, the results obtained using their methodology must be rewritten in a more convenient form. To this end, from Tables 3 and 4 , we observe that the sequence $\left\{q_{i}\right\}_{i \geq 1}$ has a sign pattern of period four $(--++)$ for $i \geq 4$. Then, taking into account this sign pattern, we enunciate the following.

Theorem 2. For any integer $m \geq 5$, there exists a positive integer $n(m)$ such that:

(i) if $q_{m-1}>0$ and $q_{m}>0$, then $\lambda_{m-1}(n)<\lambda_{n}<\lambda_{m}(n)$ for $n \geq n(m)$, and

(ii) if $q_{m-1}<0$ and $q_{m}<0$, then $\lambda_{m}(n)<\lambda_{n}<\lambda_{m-1}(n)$ for $n \geq n(m)$, where $\lambda_{m}(n):=n+2 / 3+\sum_{i=1}^{m} q_{i} / n^{i}$.

The proof of Theorem 2 follows exactly the same lines of reasoning that led to the results given in Adell and Jodrá [2]. Table 6 presents tight upper and lower bounds for $\lambda_{n}$ together with a few values $n(m)$. As we can see, the best rational bounds for $\lambda_{n}$ valid for all integers $n \geq 1$ are obtained by considering the first nine coefficients in the asymptotic expansion of $\lambda_{n}$.

Table 6. Upper and lower bounds for $\lambda_{n}$.

\begin{tabular}{|c|c|}
\hline Sharper bounds for $\lambda_{n}$ for & $n \geq n(m)$ \\
\hline$\lambda_{9}(n)<\lambda_{n}<\lambda_{8}(n)$ & $n \geq 1$ \\
\hline$\lambda_{14}(n)<\lambda_{n}<\lambda_{15}(n)$ & $n \geq 2$ \\
\hline$\lambda_{22}(n)<\lambda_{n}<\lambda_{23}(n)$ & $n \geq 3$ \\
\hline$\lambda_{29}(n)<\lambda_{n}<\lambda_{28}(n)$ & $n \geq 4$ \\
\hline$\lambda_{34}(n)<\lambda_{n}<\lambda_{35}(n)$ & $n \geq 5$ \\
\hline$\lambda_{41}(n)<\lambda_{n}<\lambda_{40}(n)$ & $n \geq 6$ \\
\hline$\lambda_{46}(n)<\lambda_{n}<\lambda_{47}(n)$ & $n \geq 7$ \\
\hline$\lambda_{53}(n)<\lambda_{n}<\lambda_{52}(n)$ & $n \geq 8$ \\
\hline$\lambda_{58}(n)<\lambda_{n}<\lambda_{59}(n)$ & $n \geq 9$ \\
\hline
\end{tabular}

Final remark. Chen and Rubin [7] conjectured that the sequence $\left\{\lambda_{n}-\right.$ $n\}_{n \geq 0}$ is strictly decreasing, which was proved by Alm [3] (cf. also Alzer [4] and Adell and Jodrá [1] for alternative proofs). In addition, Alzer [4] conjectured that the sequence $\left\{\lambda_{n}-n\right\}_{n \geq 0}$ is completely monotone and, in this respect, Alzer [5] has shown that the sequence $\left\{\lambda_{n}-n\right\}_{n \geq 0}$ is strictly convex. We recall that a sequence $\left\{\alpha_{n}\right\}_{n \geq 0}$ is said completely monotone if $(-1)^{k} \triangle^{k} \alpha_{n} \geq 0$ for $k=$ $0,1, \ldots$ and $n=0,1, \ldots$, where $\triangle^{0} \alpha_{n}:=\alpha_{n}$ and $\triangle^{k} \alpha_{n}:=\triangle^{k-1} \alpha_{n+1}-\triangle^{k-1} \alpha_{n}$ $(k=1,2, \ldots ; n=0,1, \ldots)$; in particular, the case $k=1(k=2)$ corresponds to a decreasing (convex) sequence. By using the upper and lower bounds for $\lambda_{n}$ presented in this paper, we have checked that $(-1)^{k} \triangle^{k}\left(\lambda_{n}-n\right)>0$ for $k=3,4, \ldots, 60$, which strongly suggests that Alzer's conjecture is true. 


\section{Acknowledgements}

The author expresses his sincere thanks to the anonymous referees for their insightful comments, which led to an improvement of the paper.

\section{References}

[1] J.A. Adell and P. Jodrá. Sharp estimates for the median of the $\Gamma(n+1,1)$ distribution. Statist. Probab. Lett., 71(2):185-191, 2005. http://dx.doi.org/10.1016/j.spl.2004.10.025.

[2] J.A. Adell and P. Jodrá. On a Ramanujan equation connected with the median of the Gamma distribution. Trans. Amer. Math. Soc., 360(7):3631-3644, 2008. http://dx.doi.org/10.1090/S0002-9947-07-04411-X.

[3] S.E. Alm. Monotonicity of the difference between median and mean of gamma distributions and of a related Ramanujan sequence. Bernoulli, 9(2):351-371, 2003. http://dx.doi.org/10.3150/bj/1068128981.

[4] H. Alzer. Proof of the Chen-Rubin conjecture. Proc. Roy. Soc. Edinburgh Sect. A, 135(4):677-688, 2005. http://dx.doi.org/10.1017/S0308210500004066.

[5] H. Alzer. A convexity property of the median of the gamma distribution. Statist. Probab. Lett., 76(14):1510-1513, 2006. http://dx.doi.org/10.1016/j.spl.2006.03.011.

[6] K.O. Bowman, L.R. Shenton and G. Szekeres. A Poisson sum up to the mean and a Ramanujan problem. J. Stat. Comput. Simul., 20(2):167-173, 1984. http://dx.doi.org/10.1080/00949658408810769.

[7] J. Chen and H. Rubin. Bounds for the difference between median and mean of Gamma and Poisson distributions. Statist. Probab. Lett., 4(6):281-283, 1986. http://dx.doi.org/10.1016/0167-7152(86)90044-1.

[8] T.S. Chihara. An Introduction to Orthogonal Polynomials. Gordon and Breach, New York, 1978.

[9] K.P. Choi. On the medians of Gamma distributions and an equation of Ramanujan. Proc. Amer. Math. Soc., 121(1):245-251, 1994. http://dx.doi.org/10.1090/S0002-9939-1994-1195477-8.

[10] A. Heck. Introduction to Maple, third edition. Springer-Verlag, New York, 2003.

[11] F. Jiménez and P. Jodrá. On the computer generation of the Erlang and negative binomial distributions with shape parameter equal to two. Math. Comput. Simul., 79(5):1636-1640, 2009.

http://dx.doi.org/10.1016/j.matcom.2008.07.010.

[12] J.C.W. Marsaglia. The incomplete Gamma function and Ramanujan's rational approximation to $e^{x}$. J. Statis. Comput. Simul., 24(2):163-168, 1986. http://dx.doi.org/10.1080/00949658608810899.

[13] S. Ramanujan. Question 294. J. Indian Math. Soc., 3:128, 1911.

[14] S. Ramanujan. Collected Papers. Chelsea Publishing Co., New York, 1962. 